\title{
High-Resolution In Vivo Imaging of Hippocampal Dendrites and Spines
}

\author{
Adi Mizrahi, ${ }^{\star}$ Justin C. Crowley, ${ }^{\star}$ Eran Shtoyerman, and Lawrence C. Katz \\ Howard Hughes Medical Institute and Department of Neurobiology, Duke University Medical Center, Durham, North Carolina 27710
}

Structural changes in hippocampal dendrites and dendritic spines are thought to be a consequence of a wide range of experience- and activity-dependent manipulations. We explored the dynamics of hippocampal dendritic spines in vivo by developing a surgical preparation of the adult mouse brain that enabled two-photon imaging of fluorescently labeled CA1 pyramidal neurons. Dendritic trees and spines were repeatedly visualized over many hours in exquisite detail. We tested spine stability under both control conditions and during prolonged epileptic seizures. Remarkably, spines remained structurally stable after $30 \mathrm{~min}$ of experimental induction of epileptic seizures. Spines began to disappear only several hours after induction of epileptic activity. We thus demonstrate that this technique provides a methodology for direct in vivo optical studies of the intact mammalian hippocampus.

Key words: dendrite; epilepsy; hippocampus; two-photon imaging; morphometry; seizure

\section{Introduction}

Structural changes in hippocampal dendritic spines correlate with diverse biological phenomena in adult mammals, such as the estrus cycle in female rats (Woolley et al., 1990), hibernation in squirrels (Popov and Bocharova, 1992), and enriched environments in mice (Rampon et al., 2000). Moreover, alteration in spine number is a particularly attractive cellular mechanism for encoding activity-dependant modifications in neuronal circuits (Bailey and Kandel, 1993; Yuste and Bonhoeffer, 2001).

Two-photon laser scanning microscopy (2PLSM) combined with genetic labeling of neurons has enabled repeated imaging of neurons in the intact brain. However, in mammals, imaging has been restricted to superficial layers of the brain, such as the neocortex and olfactory bulb (Grutzendler et al., 2002; Trachtenberg et al., 2002; Mizrahi and Katz, 2003); deeper subcortical structures have remained beyond the reach of current optical imaging technologies. The hippocampus in adult mice is located beneath over a millimeter of light scattering neocortex and white matter, which prevents acquiring high-resolution 2PLSM images of its neurons. Consequently, detailed time-lapse images of hippocampal neurons have only been obtained from either slice preparations or dissociated cultures. Whether pyramidal neurons in hippocampus show structural changes in the intact brain under any circumstances is currently a matter of debate.

To directly image hippocampal neurons, we developed an

Received Nov. 25, 2003; revised Nov. 25, 2003; accepted Feb. 5, 2004.

Ths work was supported by National Institutes of Health Grant DC005671. A.M. is supported by a fellowship from the International Human Frontier Science Program Organization. L.C.K. is an investigator in the Howard Hughes Medical Institute. We thank L. Belluscio for his contribution to the initial phases of this work. J. 0. McNamara and S.C. Danzer provided helpful comments. G. Feng generously provided the GFP-0 mice.

*A.M. and J.C.C contributed equally to this work.

Correspondence should be addressed to Adi Mizrahi at the above address. E-mail: mizrahi@neuro.duke.edu.

J. C. Crowley's present address: Department of Biological Sciences and Center for the Neural Basis of Cognition, Carnegie Mellon University, Pittsburgh, PA 15213.

DOI:10.1523/JNEUROSCI.5218-03.2004

Copyright $\odot 2004$ Society for Neuroscience $\quad$ 0270-6474/04/243147-05\$15.00/0 acute preparation that enabled high-resolution imaging of CA1 hippocampal neurons and dendritic spines in mice expressing green fluorescence protein (GFP) in a subset of pyramidal neurons (Feng et al., 2000) (see Fig. 1a). We focused on basal dendrites, which are located in stratum oriens, the dorsal most layer of the hippocampus.

\section{Materials and Methods}

Animals. Adult (postnatal days 55-80) transgenic mice (line GFP-O; Feng et al., 2000) were used in all experiments. Animals were maintained at the Duke University animal facility. Animal care and experiments were in accordance with the National Institutes of Health guidelines and approved by the Duke University Institutional Animal Care and Use Committee.

Surgery. Mice were anesthetized by urethane ( $1.64 \mathrm{gm} / \mathrm{kg}$ body weight, i.p., divided over three injections) and held in a custom-made stereotaxic device affixed to the microscope stage. A circular cranial window $(\sim 2.5$ $\mathrm{mm}$ in diameter) was opened in the skull (center of window, $2.5 \mathrm{~mm}$ posterior and $2.2 \mathrm{~mm}$ lateral to bregma) (supplemental Fig. 1a,b, available at www.jneurosci.org). Blunt dissection and aspiration were used to remove the cortical tissue above the hippocampus (supplemental Fig. $1 c$ ). Once the dorsal surface of the hippocampus was exposed, it was thoroughly irrigated with saline, and a cylinder cut from polyethylene tubing (outer diameter, $\sim 2.3 \mathrm{~mm}$; inner diameter, $\sim 1.6 \mathrm{~mm}$ ) was inserted into the brain as a mechanical support bridging the hippocampus, the cortex, and the cranial window (supplemental Fig. 1d). Once positioned, the cylinder was filled with $2-4 \%$ agarose (diluted in artificial $\mathrm{CSF}$ ), $\mathrm{pH} 7.4$, covered by a circular cover glass (5 mm diameter), and sealed with dental acrylic (supplemental Fig. 1e). Animals were then transferred to the stage of the 2PLSM. Body temperature was maintained, and heart rate and blood oxygenation $\left(\mathrm{spO}_{2}\right)$ were monitored. After imaging experiments, animals were killed by pentobarbital overdose.

Two-photon imaging and morphometric analysis. In vivo 2PLSM imaging was performed on a Zeiss (Oberkochen, Germany) LSM 510 equipped with a $40 \times[0.8$ numerical aperture (NA) $]$ IR-Achroplan water immersion objective and an external photomultiplier tube. A TitaniumSapphire laser (Tsunami; Spectra-Physics, Fremont, CA) pumped by a 10 $\mathrm{W}$ diode laser was used to excite GFP at $\sim 865 \mathrm{~nm}$. 
To avoid photodynamic damage, we limited our experiments to two or three time points. First, a low-magnification $\mathrm{Z}$ stack was acquired to identify cell bodies and primary dendrites as landmarks for re-identifying dendrites during the experiment. Next, three to five high-magnification image stacks $(0.7-1 \mu \mathrm{m} \mathrm{Z}$ steps) of regions of interest were acquired. Images ( $1024 \times 1024$ pixels) were acquired at 12 bits, and care was taken to adjust fluorescence levels to partial saturation of the thickest dendrites.

Movements caused by heartbeat, respiration, and slow drifts prevented online improvement of the signal-to-noise ratio by frame averaging. Instead, each region of interest was scanned at high speed 4-10 times (pixel dwell time, $0.64-0.8 \mu \mathrm{sec} /$ pixel), resulting in $4-10$ raw data image stacks. The stacks were then processed offline to correct the movement artifacts in four steps as follows. (1) To align the different stacks in three dimensions, the first stack served as the reference stack. All of the frames from the second- $n$th stacks were shifted in three dimensions to maximally match each frame within the first stack. Maximal match was defined as the maximal value of a two-dimensional normalized cross correlation between two images. (2) To correct for the fast movement artifacts within the frames (caused by the heartbeat), each frame was divided into segments composed of 10-20 consecutive lines. Here again, the first stack served as the reference. Each segment of each frame in the second- $n$th stacks was compared with the appropriate segment in the first stack by a two-dimension cross correlation and shifted to the location of the peak of the correlation. (3) Then, corresponding frames in consecutive stacks were averaged to obtain a single "average stack." (4) Averaged images were then filtered using a two-dimension low-pass filter (cutoff, 5 cycles $/ \mu \mathrm{m})$. One example of a large $(\sim 1 \mu \mathrm{m})$ movement artifact and its offline correction is shown in supplemental Figure 2.

Quantitative analyses of spines were performed manually using a program written in Matlab (MathWorks, Natick, MA) that enabled simultaneous visual comparison of two $\mathrm{Z}$ stacks. The software is designed such that a cursor appears in the two images simultaneously, enabling a more rapid and reliable spine scoring than simple visual comparison. Using this comparator software, we scored stable, new and lost spines between two time points. For analysis of spine density in fixed versus live tissue, we used the Neurolucida software (MicroBrightField, Colchester, VT). Analyses were done blind to the experimental condition and imaging order. Given the relatively poor axial resolution of optical microscopy, we could not detect small spines that projected axially, and thus we view our spine counts as a representative sample of the total pool of spines.

Induction of epileptiform activity. While under urethane anesthesia, mice were injected with a single dose of pilocarpine $(340 \mathrm{mg} / \mathrm{kg}$, i.p. $)$. Peripheral muscarinic effects were reduced by previous administration of methyl scopolamine $(1 \mathrm{mg} / \mathrm{kg}$, s.c.; $30 \mathrm{~min}$ before the pilocarpine injection). Epileptic activity was monitored by EEG recording and observation of motor responses. In some cases, an additional pilocarpine injection $(170 \mathrm{mg} / \mathrm{kg}$, i.p. $)$ was administered because a single dose did not always induce robust epileptic activity.

Bicuculline experiment. Mice were first imaged as described above. The agarose within the cranial chamber was removed, and the hippocampus was superfused with $250-500 \mu \mathrm{M}$ bicuculline for $30 \mathrm{~min}$. It was then thoroughly rinsed with saline, and a new chamber was constructed for re-imaging.

Time course of the manipulations between imaging sessions. In control experiments, imaging sessions were $4 \mathrm{hr}$ apart. In the pilocarpine experiments, mice were first imaged, injected with pilocarpine, and imaged again at least $30 \mathrm{~min}$ after epileptic activity (total time between imaging sessions, $2 \mathrm{hr}$ ). In the bicuculline experiments, mice were first imaged, treated with bicuculline for $30 \mathrm{~min}$, and re-imaged $4 \mathrm{hr}$ later (total time between imaging sessions, $\sim 5 \mathrm{hr}$ ).

Histology. Mice were perfused transcardially with $0.9 \%$ saline, followed by $4 \%$ paraformaldehyde, and the brains were prepared for histology as described by Mizrahi and Katz (2003). Brains were sliced horizontally (40 $\mu \mathrm{m}$ slices) on a sliding microtome and mounted on glass slides. Dendrites and spines were imaged with a confocal microscope (LSM510; Zeiss) using a $40 \times(1.3 \mathrm{NA})$ oil immersion objective.

\section{Results}

Because the ventral portion of the neocortex is separated from the dorsal part of the hippocampus by the intervening cingulum bundle, the neocortex (including parts of somatosensory, anteromedial visual, and primary motor cortices) can be aspirated without damaging the surface of the hippocampus. Thus exposed, we irrigated the hippocampal surface with saline, supported the surrounding cortex with polyethylene tubing enclosing a column of agarose, and sealed the craniotomy with a cover glass cemented to the skull (supplemental Fig. 1). Entire basal dendritic arbors, cell bodies, and spines in stratum oriens were imaged in detail (Fig. 1; supplemental videos 1,2 ). In some preparations, we also imaged spines on the proximal portion of apical dendrites. Unlike in vivo images obtained from mouse neocortex or olfactory bulb, which are generally extremely stable, in hippocampus a movement artifact of up to $1 \mu \mathrm{m}$, coupled to the heart beat cycle, was sometimes present. Offline image processing was used to correct these movement artifacts (supplemental Fig. 2).

We observed the complete range of spine morphologies described previously in vitro and in fixed preparations (Parnass et al., 2000; Sorra and Harris, 2000; Yuste and Bonhoeffer, 2004). Spine densities measured in vivo and in fixed tissue were indistinguishable (fixed tissue, $0.87 \pm 0.24$ spines $/ \mu \mathrm{m}, n=346$ spines; in vivo, $0.92 \pm 0.15$ spines $/ \mu \mathrm{m}, n=225$ spines; $t$ test; $p=0.51$ ). Examples of the quality of the genetic staining of the CA1 basal dendrites in the GFP-O mice are shown in supplemental Figure 3 for fixed tissue in both one- and two-photon excitation.

To assess the stability of this preparation, a spiny dendrite (within a $46 \times 46 \times 24 \mu \mathrm{m}$ region; $n=62$ spines) was imaged five consecutive times at 5 min intervals (Fig. 2), and single spines were followed over time. No loss or gain of spines was evident by any comparison between time points. This suggests that the preparation is amenable to repeated excitation, and any changes we might detect over longer time intervals are not likely to result from photodynamic damage or limitations on optical resolution.

Because of the technical challenges associated with in vivo imaging (i.e., brain pulsation and tissue-mediated light scatter) and limitations of resolving power, changes in spines shape were difficult to quantify. Thus, we operationally defined spine stability-plasticity as whether or not a spine was present throughout an experiment.

To determine structural dynamics at longer intervals, we imaged hippocampal dendritic spines 4 hr apart (Fig. $3 a$ ). Over this time period, virtually all spines $(98.6 \%)$ were stable (Table 1$)$. Although rare events of apparent spine loss and gain were observed, these may lie within the noise range of our analysis. Indeed, in our control spine population, we observed $\sim 1 \%$ spine loss and $\sim 0.3 \%$ spine gain over $4 \mathrm{hr}$. Because spine loss consistently exceeded spine gain, these minute changes most likely reflect some limitation of the preparation itself rather than a biologically significant change.

To test whether massive, synchronized activity would alter spine stability, we first used pilocarpine injections to induce hippocampal epileptic seizures while simultaneously monitoring dendritic spine morphology. Remarkably, the stability of spines was indistinguishable from the controls: over $98 \%$ of the spines remained structurally stable after $30 \mathrm{~min}$ of epileptic activity induced by pilocarpine (Fig. $3 b$, Table 1 ). For longer time points $(4$ $\mathrm{hr}$ ), we also followed spines before and after local application of bicuculline, a $\mathrm{GABA}_{\mathrm{A}}$ blocker that also elicits increased activity. 


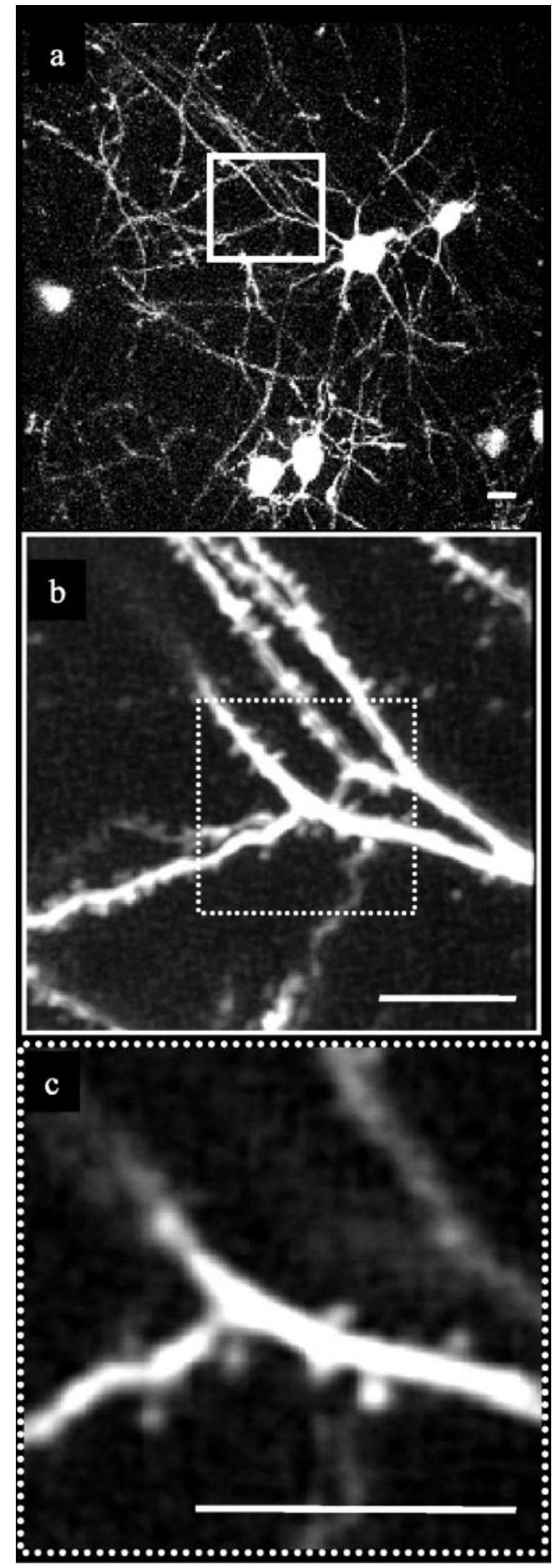

Figure 1. In vivo imaging of dendrites and dendritic spines in hippocampal CA1 neurons. $a$ In vivo image (single optical slice) of the pyramidal cell body layer observed via the imaging chamber using a $40 \times$ objective. Within the field of view, scattered pyramidal neurons, cell bodies, and basal dendrites are readily visible in stratum oriens. $b$, A projection image $(9 \mu \mathrm{m}$ in the $z$-axis) from the boxed region in $a$. The branching pattern of secondary and tertiary dendritic branches is evident, as are numerous dendritic spines. c, High magnification of a single optical slice from the in vivo preparation (from the dotted box in $b$ ). Single spines are readily discernable at high contrast. Scale bars, $10 \mu \mathrm{m}$.

Within this time frame, bicuculline induced a small increase in spine loss compared with controls (Fig. $3 c$, Table 1 ).

Thus, in a preparation of the hippocampus with intact local circuitry, spines remain morphologically stable in the face of re-

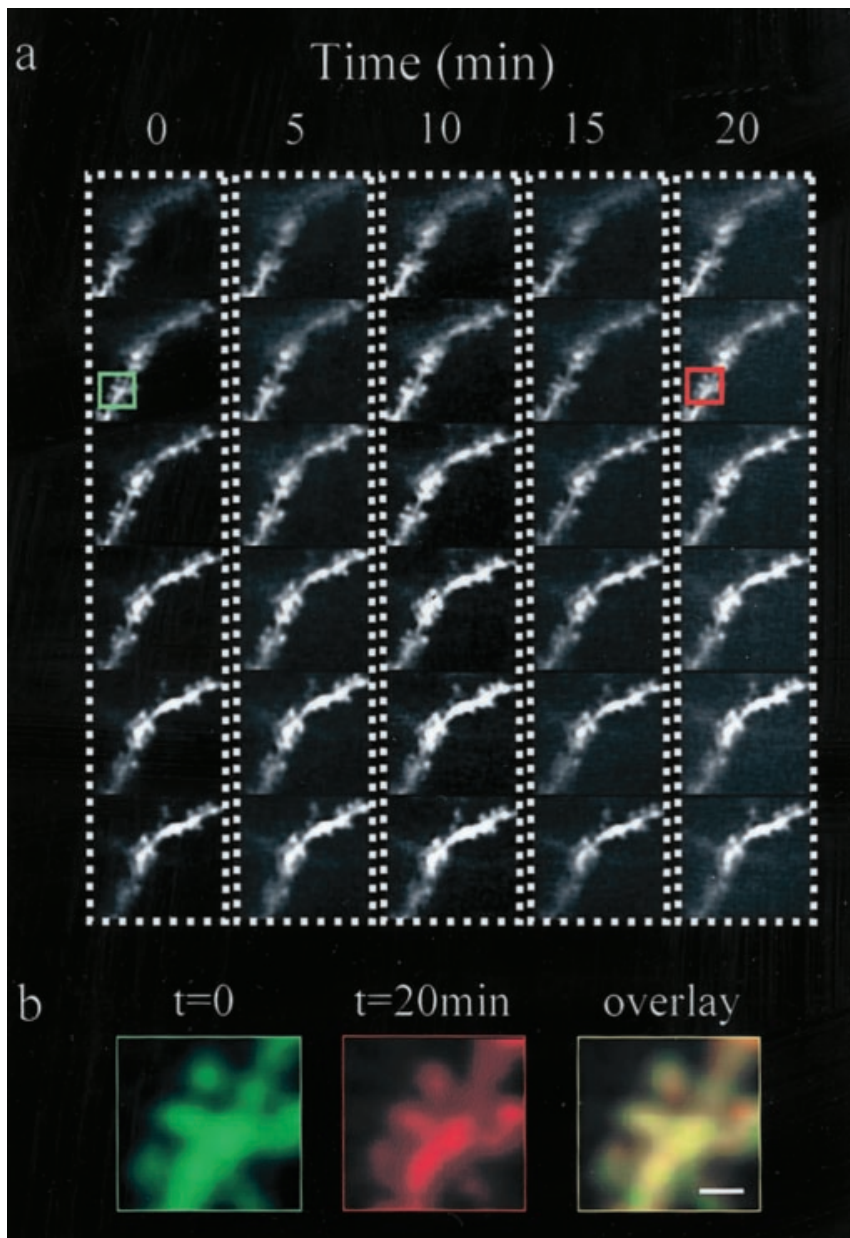

Figure 2. Stability of the preparation after repeated exposures during a 20 min interval. $a$ Representative example of a spiny CA1 dendritic segment imaged in vivo for five times during a $20 \mathrm{~min}$ period ( $5 \mathrm{~min}$ intervals). Each column is a $Z$ series composed of six images $(0.7 \mu \mathrm{m}$ intervals). $b$, High magnification of the green and red boxed region from $a$. The image on the left (pseudocolored green) shows spines at time 0 , the center image (pseudocolored red) shows the same spines at the fifth imaging session, and the image on the right is a merged image of the green and red images. Scale bar, $1 \mu \mathrm{m}$.

peated excitation. This preparation should prove useful for studies of normal and pathological conditions in which direct observation of the intact hippocampus is required.

\section{Discussion}

In vivo imaging has demonstrated that dendrites, spines, and axons in adult cortex and olfactory bulb exhibit various degrees of behaviors, including gain, loss, and shape changes (Grutzendler et al., 2002; Trachtenberg et al., 2002; Mizrahi and Katz, 2003). If we assume no noise in our sample of $\sim 1200$ spines, we would be able to detect a minimum turnover rate of $0.5 \%$ per day. Because even the highest estimates of flux in cortex range from $\sim 0.5$ to $1 \%$ change per day (Trachtenberg et al., 2002), it is perhaps not surprising that hippocampal spines remained stable over $4 \mathrm{hr}$. Nevertheless, we determined that, in the intact adult hippocampus, there is unlikely to be a rapid rate $(>0.5 \%$ per day) of de novo spine formation and spine retraction.

Several studies have directly shown spinogenesis in hippocampal neurons accompanying long-term synaptic change (Engert and Bonhoeffer, 1999; Maletic-Savatic et al., 1999). Indeed, the emerging consensus is that efficacy changes in synapses are accompanied by structural changes in dendritic spines (Yuste 


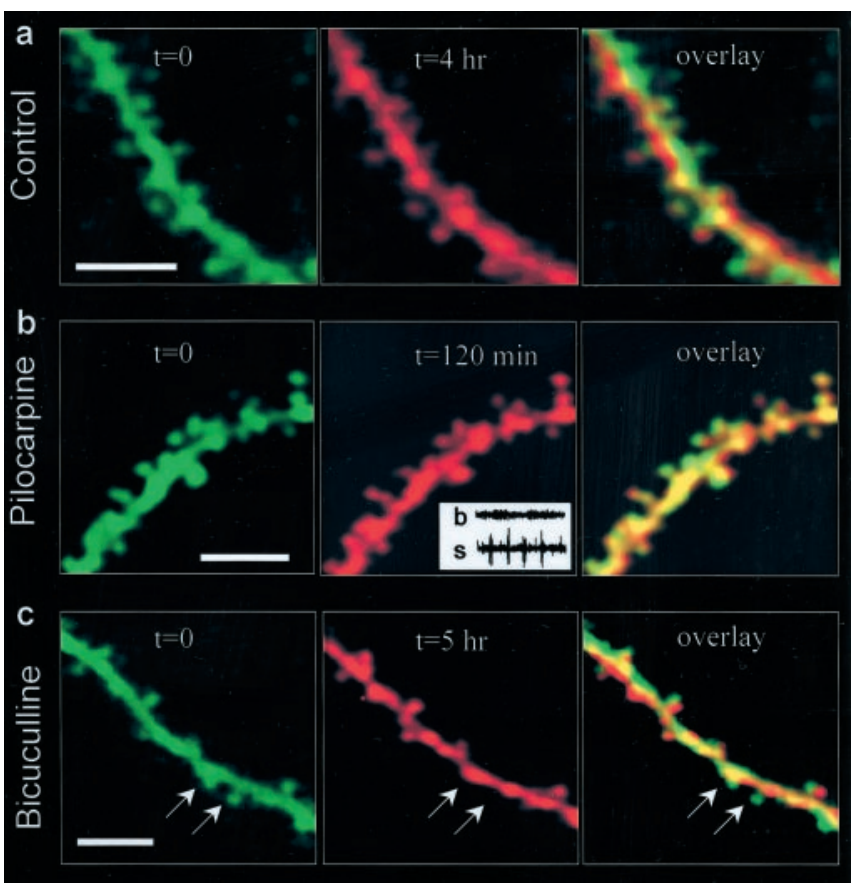

Figure 3. Time-lapse imaging of spines in CA1 hippocampal neurons in vivo. $a-c$, Representative examples of spiny dendrites from CA1 basal dendrites under control conditions $(a)$, during pilocarpine-induced seizures $(b)$, and after application of bicuculline $(c)$. The first imaging session ( $t=0$, pseudocolored green) is compared with the second imaging session (pseudocolored red) by overlying the two images (overlay). The overlays indicate little change in spines. However, rare instances of spine loss were observed after bicuculline treatment (arrows in $c$ ). Inset in $b, 10$ sec traces from the EEG recordings before $(b)$ and during the seizure $(s)$. All images are single optical slices. Scale bars, $5 \mu \mathrm{m}$.

Table 1. Stability of spines in hippocampal neurons

\begin{tabular}{llllll}
\hline Experiment & $\begin{array}{l}\text { Spines } \\
\text { (total) }\end{array}$ & $\begin{array}{l}\text { Stable spines } \\
(\%)\end{array}$ & $\begin{array}{l}\text { New spines } \\
(\%)\end{array}$ & $\begin{array}{l}\text { Lost spines } \\
(\%)\end{array}$ & $n$ \\
\hline $\begin{array}{c}\text { Control } \\
\quad(4 \mathrm{hr} \text { apart) }\end{array}$ & 1286 & $1268(98.6)$ & $4(0.3)$ & $15(1.1)$ & 4 \\
$\begin{array}{c}\text { Pilocarpine } \\
(2 \mathrm{hr} \text { apart) }\end{array}$ & 680 & $667(98.1)$ & $1(0.1)$ & $12(1.8)$ & 2 \\
$\begin{array}{c}\text { Bicuculline } \\
(5 \mathrm{hr} \text { apart) }\end{array}$ & 412 & $392(95.1)$ & 0.0 & $20(4.9)$ & 2 \\
\hline
\end{tabular}

*For detailed description of the manipulations and imaging intervals, see Materials and Methods.

and Bonhoeffer, 2001,2004; Nikonenko et al., 2002) (but see Sorra and Harris, 2000). Direct evidence of spinogenesis from the hippocampus of adult animals is scarce, and it seems that only massive alterations in activity, such as global silencing, activation, or acute slice preparation, can induce structural changes (Kirov et al., 1999).

Although numerous neurological conditions are associated with various forms of altered spine number and morphologies (Fiala et al., 2002), there is little direct evidence suggesting that these alterations are a direct consequence of changes in activity levels, because activity is only one variable in the complex pathology of any disease or injury. Spine changes may well be attributable to an indirect effect of neurodegenerative processes triggered by recurrent epileptic activity. We showed that spines on hippocampal neurons in vivo remain stable in the short term, despite the massively correlated waves of activity present during seizure events (i.e., pilocarpine-induced seizures). Spine loss started to appear only after several hours of induced seizures (after bicuculline application).
Although an exposed hippocampus preparation was used for electrophysiological studies over 40 years ago (Kandel et al., 1961), the morphology of hippocampal neurons has only been studied at high resolution in fixed tissue and in vitro. The in vivo preparation described here completely preserves the normal circuitry of hippocampal neurons and time-lapse (repeated measures) analyses are possible for several hours. One example of a spiny dendrite imaged over $8 \mathrm{hr}$ is shown in supplemental Figure 4. These data demonstrate the stability of the preparation for twice the duration used in our control experiments data. We believe that imaging intervals much longer than $8 \mathrm{hr}$ will be challenging because of the time limitations associated with the urethane anesthetic (e.g., the long-term, deleterious effects of urethane on organs). Urethane produces long-term, surgical anesthesia without dramatically suppressing neural activity or responsiveness and does not prevent seizure induction. However, the pharmacology of urethane, including its potential effects on NMDA receptors, is a potential confound that may cause us to underestimate the dynamics of spines in unanesthetized animals. Whether high-resolution imaging of the hippocampus is successful under different conditions remain to be investigated.

In part because in vivo imaging of neurons in superficial layers of the brain is uncomplicated by light scattering and absorption, most in vivo imaging techniques have focused on the dorsal surface of the brain and primarily the most dorsal layers of the cortex. The preparation described here provides a window to image the hippocampus not only with 2PLSM but also with other functional techniques, including optical imaging of intrinsic signals, calcium-sensitive indicators, and voltage-sensitive dyes.

Note added in proof. In a paper appearing after this manuscript was accepted, Levene et al. (2004) describe the use of grin lenses based on two-photon imaging to visualize hippocampal neurons in vivo.

\section{References}

Bailey CH, Kandel ER (1993) Structural changes accompanying memory storage. Annu Rev Physiol 55:397-426.

Engert F, Bonhoeffer T (1999) Dendritic spine changes associated with hippocampal long-term synaptic plasticity. Nature 399:66-70.

Feng G, Mellor RH, Bernstein M, Keller-Peck C, Nguyen QT, Wallace M, Nerbonne JM, Lichtman JW, Sanes JR (2000) Imaging neuronal subsets in transgenic mice expressing multiple spectral variants of GFP. Neuron 28:41-51.

Fiala JC, Spacek J, Harris KM (2002) Dendritic spine pathology: cause or consequence of neurological disorders? Brain Res Rev 39:29-54.

Grutzendler J, Kasthuri N, Gan WB (2002) Long-term dendritic spine stability in the adult cortex. Nature 420:812-816.

Kandel ER, Spencer WA, Brinley Jr FJ (1961) Electrophysiology of hippocampal neurons. I. Sequential invasion and synaptic organization. J Neurophysiol 24:225-242.

Kirov SA, Sorra KE, Harris KM (1999) Slices have more synapses than perfusion-fixed hippocampus from both young and mature rats. J Neurosci 19:2876-2886.

Levene MJ, Dombeck DA, Kasischke, Molloy RP, Webb WW (2004) In vivo multiphoton microscopy of deep brain tissue. J Neurophysiol 91:1908 1912.

Maletic-Savatic M, Malinow R, Svoboda K (1999) Rapid dendritic morphogenesis in CA1 hippocampal dendrites induced by synaptic activity. Science 283:1923-1927.

Mizrahi A, Katz LC (2003) Dendritic stability in the adult olfactory bulb. Nat Neurosci 6:1201-1207. 
Nikonenko I, Jourdain P, Alberi S, Toni N, Muller D (2002) Activityinduced changes of spine morphology. Hippocampus 12:585-591.

Parnass Z, Tashiro A, Yuste R (2000) Analysis of spine morphological plasticity in developing hippocampal pyramidal neurons. Hippocampus 10:561-568.

Popov VI, Bocharova LS (1992) Hibernation-induced structural changes in synaptic contacts between mossy fibers and hippocampal pyramidal neurons. Neuroscience 48:53-62.

Rampon C, Tang YP, Goodhouse J, Shimizu E, Kyin M, Tsien JZ (2000) Enrichment induces structural changes and recovery from nonspatial memory deficits in CA1 NMDAR1-knockout mice. Nat Neurosci 3:238-244.

Sorra KE, Harris KM (2000) Overview on the structure, composition, func- tion, development, and plasticity of hippocampal dendritic spines. Hippocampus 10:501-511.

Trachtenberg JT, Chen BE, Knott GW, Feng G, Sanes JR, Welker E, Svoboda $\mathrm{K}$ (2002) Long-term in vivo imaging of experience-dependent synaptic plasticity in adult cortex. Nature 420:788-794.

Woolley CS, Gould E, Frankfurt M, McEwen BS (1990) Naturally occurring fluctuation in dendritic spine density on adult hippocampal pyramidal neurons. J Neurosci 10:4035-4039.

Yuste R, Bonhoeffer T (2001) Morphological changes in dendritic spines associated with long-term synaptic plasticity. Annu Rev Neurosci 24:1071-1089.

Yuste R, Bonhoeffer T (2004) Genesis of dendritic spines: insights from ultrastructural and imaging studies. Nat Rev Neurosci 5:24-34. 